\title{
Lessons Learned Applying Commercial Off-the-Shelf Products
}

\author{
Manufacturing Resource Planning II Program
}

Lisa Brownsword

Patrick Place

June 2000

COTS-Based Systems Initiative

Unlimited distribution subject to the copyright.

Technical Note

CMU/SEI-99-TN-015 
The Software Engineering Institute is a federally funded research and development center sponsored by the U.S. Department of Defense.

Copyright 2000 by Carnegie Mellon University.

NO WARRANTY

THIS CARNEGIE MELLON UNIVERSITY AND SOFTWARE ENGINEERING INSTITUTE MATERIAL IS FURNISHED ON AN "AS-IS" BASIS. CARNEGIE MELLON UNIVERSITY MAKES NO WARRANTIES OF ANY KIND, EITHER EXPRESSED OR IMPLIED, AS TO ANY MATTER INCLUDING, BUT NOT LIMITED TO, WARRANTY OF FITNESS FOR PURPOSE OR MERCHANTABILITY, EXCLUSIVITY, OR RESULTS OBTAINED FROM USE OF THE MATERIAL. CARNEGIE MELLON UNIVERSITY DOES NOT MAKE ANY WARRANTY OF ANY KIND WITH RESPECT TO FREEDOM FROM PATENT, TRADEMARK, OR COPYRIGHT INFRINGEMENT.

Use of any trademarks in this report is not intended in any way to infringe on the rights of the trademark holder.

Internal use. Permission to reproduce this document and to prepare derivative works from this document for internal use is granted, provided the copyright and "No Warranty" statements are included with all reproductions and derivative works.

External use. Requests for permission to reproduce this document or prepare derivative works of this document for external and commercial use should be addressed to the SEI Licensing Agent.

This work was created in the performance of Federal Government Contract Number F19628-95-C-0003 with Carnegie Mellon University for the operation of the Software Engineering Institute, a federally funded research and development center. The Government of the United States has a royalty-free government-purpose license to use, duplicate, or disclose the work, in whole or in part and in any manner, and to have or permit others to do so, for government purposes pursuant to the copyright license under the clause at 52.227-7013.

For information about purchasing paper copies of SEI reports, please visit the publications portion of our Web site (http://www.sei.cmu.edu/publications/pubweb.html). 


\section{Contents}

1 Introduction 1

1.1 COTS Terminology 2

1.2 Program's Background 3

1.3 Program's COTS Approach 4

1.4 Review Process 5

1.5 Acknowledgements 6

2 Lessons Learned $\quad 7$

2.1 Lessons on Business Processes 8

2.2 Lessons on Product Evaluation and Acquisition 10

2.3 Lessons on Programmatic Issues 13

2.4 Lessons on Transition Issues 14

2.5 Lessons on User Communities 16

2.6 Lessons on Deployment 17

2.7 Lessons on Vendor Relationship Management 19

3 General Conclusions 20

For Additional Information 21 


\section{Abstract}

While the lure of easy system construction from pre-existing building blocks that snap into place is appealing, current reality reveals a less than ideal picture, particularly for commercial off-the-shelf (COTS) software components. Examining the similarities and differences of organizations that have applied COTS and the successes and failures of those organizations has enabled the COTS-Based Systems (CBS) Initiative at the Software Engineering Institute (SEI) to identify a number of significant capabilities that an organization must have to succeed with a COTS-based approach. This case study of the Manufacturing Resource Planning II program is part of a series of case studies that seek to identify important acquisition, business, and engineering issues surrounding the use of COTS-based systems and thus derive available solutions, where possible. 


\section{Introduction}

The use of existing "off-the-shelf" (OTS) products to produce systems is accelerating. Interest in applying a full or partial OTS solution is fueled by the need of organizations to acquire functioning systems faster and, potentially, at a reduced cost. This can be an effective long-term approach to system development, maintenance, and reengineering.

While the lure of easy system construction from pre-existing building blocks that snap into place is appealing, current reality reveals a less than ideal picture, particularly for commercial OTS (COTS) software products. Available COTS software seldom fits harmoniously with other system components without some amount of adaptation to make all the components work together. Currently, there is limited experience and available guidance on how to effectively approach the acquisition and maintenance of software-intensive systems built using COTS software.

The COTS-Based Systems (CBS) Initiative is one of the technical engineering practice initiatives at the Software Engineering Institute (SEI) and is aimed at establishing and demonstrating principles and practices for integrating and evolving systems from previouslybuilt and COTS products. This includes methods for evaluating the suitability and quality of products, methods for integrating products into operational systems, and acquisition and business practices that permit the effective use of CBS engineering practices.

Examining the similarities and differences of organizations that have applied COTS and the successes and failures of those organizations has enabled us to identify a number of significant capabilities that an organization must have to succeed with a COTS-based approach. This case study lists lessons learned from one, successful, Department of Defense (DoD) acquisition of a COTS-based system.

This report briefly describes the program and the interview process. Then, the lessons learned by the program are discussed; these lessons have been grouped into seven categories. Finally, some general conclusions are drawn concerning this acquisition. 


\subsection{Cots Terminology}

The terms OTS and COTS are often used to refer to products and services other than software. Although the focus of the CBS Initiative is on the use of software products in softwareintensive systems, we are finding that many of the issues uncovered through the case studies are applicable to hardware.

In our usage COTS-based systems are composed of software components that

- $\quad$ are ready and "off-the-shelf," with a predominance from a commercial source (COTS). Government off-the-shelf (GOTS), non-developmental items (NDI), or components reused from a legacy system may be included.

- have significant functionality and complexity

- $\quad$ are self-contained and possibly execute independently

- are generally used "as is" rather than requiring internal modification

- may be integrated with other components to achieve required system functionality

There are different types of COTS-based systems, leading to different implications for business, engineering, and management practices.

At one end of the CBS spectrum are COTS-solution systems. These systems comprise a single product or product suite, provided one vendor, that may be tailored to provide the system's functionality. The amount of tailoring, data conversion, and business practice reengineering is often significant. These systems may be found in application areas with general concurrence on application practices, examples being personnel management and financial management applications. PeopleSoft and Oracle are typical vendors.

At the other end of the CBS spectrum are COTS-aggregate systems. These systems are composed of many products, usually from different vendors, and other OTS components that are integrated to provide the system's functionality. Often the use of the particular set of components is unprecedented and requires substantial resources to select and integrate a cohesive set of components. These systems may be found where the needs of the system cannot be satisfied by a single product or product suite or when the system's operational procedures are unique. 


\subsection{Program's Background}

The Manufacturing Resource Planning (MRP) II program has the same name as the business practice approach. To avoid confusion throughout this report, we will distinguish between the MRP II program itself and the MRP II philosophy.

The MRP II program is responsible for the acquisition and installation of a system to perform the business functions for maintenance depots of the DoD in all services. The system chosen embodies the MRP II philosophy, which is an extension of, and controls more of the manufacturing business than, the earlier Material Requirements Planning process. The MRP II philosophy is an established domain of manufacturing practices with established standards and professional groups. The MRP II program selected the CompassCONTRACT product suite from Western Data Systems Inc. (WDS) ${ }^{1}$.

The typical business of the maintenance depots is to take a DoD asset, such as a helicopter or tank, strip it down to its carcass, and then rebuild the asset. The parts used to rebuild the asset may be those that came with it if they are undamaged. They could also be newly manufactured or repaired parts. The overall result is that the asset undergoes routine but large-scale maintenance, where every part of the asset is checked thoroughly for flaws and repaired if necessary.

Depot maintenance benefits from the MRP II philosophy, as it is statistically predictable. Statistically, maintenance of one asset requires the same level of effort and replacement parts as the next. If the parts and labor are known to be available before the arrival of the asset, the entire maintenance effort may be performed as efficiently as possible. The examination of records determines a statistical baseline that may be adjusted as data is collected in subsequent maintenance.

The MRP II program is part of an overall strategy to maximize the commonality of business processes across all depots for all of the services. The MRP II program automates a significant piece of the depot's business systems. In part, the MRP II program is the result of the U.S. Air Force's Depot Maintenance Management Information System (DMMIS), that attempted to bring together the best components from each depot's suite of business systems to create a single depot management system. The DMMIS program, which was based on different custom software and modified COTS products, was unsuccessful, and the Joint Logistics Systems Center was tasked to provide a new system, resulting in the MRP II program.

1 It should be noted that WDS integrates COTS products into CompassCONTRACT. The underlying database is from Oracle Corporation, and one component of the suite comes from another vendor. 


\subsection{Program's COTS Approach}

The MRP II program began in early 1995. Prior to starting any source-selection activities, the program conducted a three-month COTS feasibility study followed by a three-month requirement definition effort. Source selection began with the Commerce Business Daily (CBD) announcement in the fall of 1995. A fixed-price contract was awarded to WDS in the fall of 1996 with the contract starting in early 1997. The initial pilot site installation began in mid 1997. The WDS product, like many COTS-solution systems, required substantial attention to business process reengineering and data conversion for effective deployment. As such, the program's emphasis at this point focused on determining effective transition guidance for fielding into DoD depots. Additional site installation to early adopters began in the fall of 1997.

The MRP II program continues to deploy the system. Depots are in various stages of transition ranging from full implementation (when cut-over from legacy systems is complete) to early training and migration planning. Depot success with the MRP II philosophy and WDS product has also varied. Successful depots have generally seen the MRP II philosophy as central to their own success, understood that some amount of business process reengineering is required to leverage the business approach (and the WDS product), and exhibited strong leadership throughout all transition phases.

We would characterize the MRP II program as a COTS-solution system since the WDS product suite forms the system for the MRP II program. The essential elements of the program's COTS approach can be summarized as follows:

- The government would adopt commercial practices that were not in direct conflict with DoD regulations.

- The government would lead a cross-service requirement effort augmented with industry experts in the area of the MRP II philosophy.

- The new system, provided by the MRP II program, would not attempt to perform all depot management functions. Rather, it would perform a partial, but significant, set of common, core functions.

- No modification of the COTS product suite's software to create government-specific features would be allowed.

- The government would take responsibility for the selection of the COTS product suite.

- The MRP II program office would take responsibility for installing the software at the various depots and providing seed money to assist the depots in the deployment of the MRP II philosophy at their sites.

- Each depot would be responsible for determining gaps between its business processes and those supported by the selected product, performing any necessary business process reengineering, developing and managing the necessary site transition, and providing any necessary integration with other systems at its site. Additionally, each depot was responsible for providing any contractor support for these activities. 
- The MRP II program office would establish and maintain an active presence within the manufacturing resource planning community by participating in the vendor's user group, professional groups, and standards organizations.

- The MRP II program office would establish and maintain a long-term partnership with the selected COTS vendor.

\subsection{Review Process}

The MRP II review was conducted by holding an initial meeting, preparing for the review, performing the review, and analyzing the data.

The initial meeting was held via teleconference in January 1998. Participants were the MRP II program manager, the MRP II program office support contractor, and the SEI. The purpose of the meeting was to lay the foundation for the review and help the SEI understand the status and scope of the MRP II program. As a result of the meeting, the review was structured into two parts. The first part of the review would consist of a visit with the program office; the second part would involve visiting one or more depots. Actual sites would be selected during the program office visit.

SEI pre-review preparation involved the development of a questionnaire as well as gaining a better understanding of politically sensitive issues and the purpose and products of the review.

The program office review was conducted over two days in February 1998 at the MRP II program office. The SEI team consisted of two senior staff members from the CBS Initiative. Due to the small size of the program office, interviews were conducted in a group setting. The interview participants included the MRP II program manager, the deputy MRP II program manager, and several contractor staff members who had provided program office support from source selection to the present.

At the completion of the interviews, the status of each deployed site was discussed. The sites selected for inclusion in the SEI review were the Air Force Aerospace Maintenance and Regeneration Center (AMARC) in Tucson, AZ and the Naval Aviation Depot (NADEP) at Cherry Point, NC. Both sites were sufficiently far along in the deployment of MRP II to provide useful data. The SEI team for the deployed site reviews would remain the same. The deputy program manager from the MRP II program office would accompany the SEI team to provide any necessary context.

The review of AMARC was conducted during a one-day site visit in February 1998. The interview participants included the program director responsible for the transition to MRP II, the contractor project lead providing transition support to AMARC, and the technical consultant providing specific assistance in mapping the MRP II philosophy and the WDS product to the specific business environment at AMARC. 
The review of the NADEP, Cherry Point was conducted in a one-day site visit in March 1998. The interview participants included the commanding officer, the executive officer, and the MRP II site lead.

The next steps were to analyze the collected data from all the site visits, develop a detailed set of findings, and document the lessons learned. Section 2 records the lessons learned and their associated findings.

\subsection{Acknowledgements}

The SEI would like to thank the MRP II program office staff for their willingness to participate in and support this review. Their assistance, particularly in arranging site visits, and their subsequent reviews of this document have been of great value to us. 


\section{Lessons Learned}

The MRP II lessons learned are organized into the following categories:

- business processes

- product evaluation and acquisition

- programmatic issues

- transition issues

- user communities

- deployment

- vendor relationship management

No attempt was made to collect the data in the above categories or order. The organization arose during the detailed analysis of the raw data and also due to similarities with other reviews. Some lessons were derived from a single interview and other lessons were derived from multiple interviews. However, no attempt has been made to sort the lessons according to this criterion. 


\subsection{Lessons on Business Processes}

The lessons learned about business processes are discussed below.

\subsubsection{Operating in a commercial manner allows a greater use of COTS products.}

If a DoD organization is to purchase a COTS-based system, it must be prepared to operate in a more commercial manner. All software packages include assumptions about the way in which they will be used; violating these assumptions means that the software will be hard to use. In the case of the MRP II program, the maintenance depots have shown that it is possible to adapt their business processes to those of the commercial world. Because the depots were willing to change their business processes, the program office was able to purchase COTS software supporting the remanufacturing business rather than having to develop and maintain a customized system.

\subsubsection{Business processes should be reengineered before and during automation.}

The MRP II program office performed an initial examination of the depots' business practices and provided a general notion of how they would be changed. Each depot subsequently performed a more rigorous examination of its specific practices, determining how it could benefit from the WDS product.

Software, whether COTS or customized, does not change or improve the efficiency of an organization's business processes; it merely automates those processes. A COTS business product embodies a set of business practices that may be different than those used by the receiving organization. Thus, deploying a COTS product to automate the business is likely to require some changes to the organization's business practices.

The introduction of software should be delayed until the process of reengineering the business processes has been initiated. However, this reengineering cannot take place in a vacuum; it must take into account the availability of products to support the new practices.

\subsubsection{A COTS product can act as a catalyst for improving business processes.}

The MRP II program shows that the introduction of software can act as a catalyst, helping an organization examine business processes for any deficiencies and improving those processes. The maintenance depots would have gained benefits simply by improving their processes. However, optimizing their business processes to take advantage of the WDS product provided greater gain. 
The examination of business processes embodied by COTS software enables DoD organizations to gain some insight into the business processes of the commercial world and adopt those processes that make sense in their environment.

\subsubsection{It is easy to underestimate the time it takes to realistically understand and convert to new business processes.}

The introduction of the WDS product forced each depot to examine its business practices in order to use the software. The MRP II program underestimated the amount of effort it takes to understand the existing business processes within a depot, believing that the depots could adapt to the MRP II philosophy faster than industry. Evidence from industry indicates that the average length of time to transition to the MRP II philosophy is 18 months, yet the DoD estimated it could make the transition in 12 . There is no real reason to believe that the DoD can adapt to new processes faster than industry, and the evidence in this case suggests that the conversion time was similar to the industry norms.

\subsubsection{The decision to use a COTS product may require a change in policy or in business processes outside of a program.}

The choice to acquire a COTS-based system that embodies a particular set of business practices may have ramifications outside a DoD program. In particular, for the MRP II program, when a depot chooses to use the WDS product to report financial information to its commands, those commands, and possibly the DoD as a whole, must approve the new reports. 


\subsection{Lessons on Product Evaluation and Acquisition}

The lessons learned about product evaluation and acquisition are discussed below.

\subsubsection{Understanding the marketplace in relation to your own business is vital.}

One of the most important aspects of the MRP II program was the study of the marketplace to see what products were available that supported the MRP II philosophy. This study enabled the MRP II program team to shape its requirements so that COTS software could be acquired. If the DoD (or any organization) creates requirements without examining and taking into account the products in the marketplace, it is unlikely that those requirements can be met by commercial software.

\subsubsection{Create a representative team of stakeholders to shape the requirements.}

Many individuals with different functions use the business system employed by the depot. It is important to use a representative sample of the workforce to develop the requirements so that no important aspect of the overall function of the depot will be omitted. In addition, for the DoD, it is important that these representatives come from each of the services, as each service is likely to have different ways of performing similar functions. The representatives must be truly representative and, ideally, be able to speak for others with similar functions.

\subsubsection{Use outside experts and vendors to help shape the requirements.}

During requirements definition, the MRP II program employed external experts who understood the ramifications of adopting the MRP II philosophy. These experts were able to assist the program office by setting expectations as to what the MRP II philosophy could and could not do and by providing information about the products in the marketplace. Typically, users of a legacy system will create requirements that ensure that a new system performs in much the same way as their existing system. External experts will question those legacy requirements, and such questioning allows the team to define the requirements more successfully. Given that requirements are crucial for every acquisition, using all of the available expertise will improve the quality of the requirements. This leads to a more successful adoption of a new system. Poorly defined requirements could have led to a system that was hard (or worse, impossible) to use, or to a custom solution with none of the benefits of the COTS market. 


\subsubsection{Pare down the requirements to reflect your essential business process elements.}

The MRP II requirements definition team did an excellent job of understanding all of the requirements and sorting them into categories of "must have," "should have," and "nice to have." When the DoD decided that it could alter business processes, many "legacy" requirements became moot or entered the category of "nice to have." The requirements in the "must have" category are those that embody the fundamental business processes of the depots; all other requirements are negotiable. In the world of COTS software acquisition, creating a requirement solely to satisfy an existing process is insufficient. There must be a strong reason for the requirement to exist; otherwise it should be considered a preference that may be negotiated away.

\subsubsection{Use the requirements definition team for source selection.}

The MRP II program used the team that defined the requirements to perform the sourceselection process. This meant that all of the team's expertise and understanding of the needs for an MRP II system could be reused in source selection. More importantly, the benefits of having a representative team and outside experts are realized at both source selection and requirements definition. An added benefit of using the same team is that potential misunderstandings of the requirements can be avoided. Including representatives of end users in the team brings a practical aspect to source selection — the users can actually determine if the proposals meet their day-to-day needs.

\subsubsection{The use of COTS products may reduce requirements creep.}

Every long-lived system changes over time in order to meet changing or extended mission needs. However, for custom-developed systems where the DoD controls the source code, there is a danger of superfluous requirements being added, leading to requirements creep. Although the DoD may request additional features from the vendor when the system is a COTS product, those features will be added only if they make commercial sense to the vendor. The program office, by sticking to a COTS software approach of no code modification and no vendor custom engineering, can reduce the tendency for requirements creep from the depots. The depots have to accept that the COTS product operates in a particular way and that the DoD cannot control the way the product operates - that is in the purview of WDS.

\subsubsection{Different selection criteria are needed when acquiring COTS- based systems.}

The criteria used for source selection were weighted in accord with a traditional practice, which is targeted towards developmental systems rather than for the acquisition of a COTSbased system. This meant that not enough weight was given to the demonstration of the operational capabilities or the recommendations from other customers of the product. Different types of systems may require different weightings. In the case of the MRP II 
program where a single COTS product was being acquired, more weight could have been given to product demonstrations and the way that industry used the product.

\subsubsection{The selection of COTS products with considerations for ease of integration with other COTS products in an enterprise can increase the effectiveness of your investment dollars.}

Due to the application programming interfaces of the WDS product, the MRP II program has been able to connect the WDS product with the Oracle Financial product at a pilot site, providing greater capability to the enterprise.

\subsubsection{Use contracts that provide flexibility for needs identified after contract award.}

One of the biggest problems for the MRP II program office was the rigidity of the contract. Part way into the contract, the need for additional education, training, and consulting became apparent. However, there was no way in the WDS contract to add this extra support.

\subsubsection{Choosing an appropriate vendor is important to success.}

When acquiring a COTS-solution system, a DoD organization needs to consider vendor characteristics (such as stability or willingness to work with the DoD) as part of the acquisition. Although there are many companies selling products to support the MRP II philosophy, when it comes to applying MRP II principles to remanufacturing, there are a limited number of products available. WDS, the vendor that was awarded the contract, is a small, but well-established company, that had been selling an MRP II product for about 10 years. The company's customer support appears to be excellent, in that it continues to support customers using significantly older versions of the company's product as well as the customers using the latest version.

The most important aspect of WDS, however, is that it is willing to work with the DoD rather than for the DoD. It has maintained its autonomy and listened to the DoD requests for enhancements rather than automatically accepting that such changes must be made. 


\subsection{Lessons on Programmatic Issues}

The lessons learned about programmatic issues are discussed below.

\subsubsection{Contract delivery requirements lists (CDRLs) should be appropriate to the commercial marketplace.}

A result of purchasing a COTS-based system rather than developing a customized system is that the nature of contract deliverables changes. When a system is being developed, the DoD may legitimately require deliverables with respect to the documentation of the design and implementation. However, when considering a COTS product, such information belongs to the vendor and may represent its intellectual property or reflect its software development practices. There is certainly no need for this information to be delivered to the DoD. The CDRLs for the MRP II program are the vendor's release notes. This satisfies contracting requirements and allows the vendor to operate in a way that makes sense to its commercial concerns.

\subsubsection{DoD regulations at the time of this program's inception were not helpful in acquiring a COTS product.}

The regulations for system acquisition (DoD Instruction 5000.2, Defense Acquisition Management Policies and Procedures) under which the MRP II program had to operate, were defined in accord with traditional DoD practice (custom development of new systems). When applying these regulations to the acquisition of a COTS product, the MRP II program found that the regulations were not always appropriate. Typical differences included the amount of detail in the definition and flexibility of the requirements, and the different documentation needs. 


\subsection{Lessons on Transition Issues}

The lessons discussed below concern one aspect of transition -- changing the culture in the receiving organizations.

\subsubsection{If business practice changes are required to use a COTS- based system, more than the usual level of education and training is required.}

Education and training play an important role in the introduction of any new system. In the case of the MRP II program, where a new way of business was being introduced to the depots, early education would have helped the requirements definition and source-selection teams understand the new approach to business. Also, early education would have helped the depots understand how they could change their business processes to match those of the MRP II philosophy. Overall, insufficient resources were allocated for educating the depots about the MRP II philosophy.

\subsubsection{Early and creative uses of education and training are effective ways to achieve buy-in.}

One of the most important factors for the success of a program is the buy-in from all stakeholders-regardless of whether it's COTS-based or a custom-developed system. People using an existing system will generally want any new system to operate in the same way. Fear of change leads to resistance to the new system, which can be insidious and hard to overcome. But, such resistance must be overcome for the introduction of the new system to be successful. Some of the successful MRP II deployed sites have used education and training in creative ways. For example, the NADEP, Cherry Point focuses some of its early training on showing its staff that they can do their jobs better using the new business processes than they could using their existing processes. Generally, people want to perform their jobs as well as possible, so all they may need for acceptance is to be shown that adopting the new processes will help them do their jobs better.

Another approach used for applying education and training to overcome resistance was to focus training on understanding the MRP II philosophy and act as a vehicle for resolving concerns as to how various stakeholders' jobs would be affected. In some cases, education has converted detractors into advocates who have then gone on to actively promote the use of the MRP II philosophy.

\subsubsection{Investment in stakeholder education before deployment can decrease the time to deploy a new system.}

The NADEP, Cherry Point delayed deployment as long as possible, using resources for education before deployment. Therefore, the staff responsible for deploying the system at 
Cherry Point has a deeper understanding of the MRP II philosophy and has made more rapid progress toward full deployment than most of the other sites.

\subsubsection{Training oriented toward specific classes of users optimizes the transition.}

Training in the use of a COTS-based system is just as necessary as for custom-developed systems. COTS vendors vary greatly in the depth and breadth of their training materials and, to meet the demands of potentially varied end-user communities, vendor training is typically quite general. Some of the MRP II deployed sites found the vendor-provided training to be too broad and inapplicable, and not of sufficient depth for some users. Without training targeted toward different types of users, there is a danger that general training will lead to users applying the products in unexpected and possibly inefficient ways. Training targeted toward a specific class of user will help users in that class to understand the optimal way to use the system for their particular job. Program staff members should consider the need for creating specialized training for their organization. Some vendors may provide customized training at an additional expense, or independent consultants may be able to provide focused training.

\subsubsection{The high motivation of the depots to stay competitive with the commercial repair and remanufacturing world can greatly aid in the transition to a new system.}

Many of the maintenance depots were highly motivated to improve their business practices. The MRP II program may be unusual in that the clients of the program office (the depots) have a great desire to improve the way they do business. The depots realize that their business is repairing DoD assets; they also realize that there are commercial companies capable of repairing those same assets. If the depots cannot compete with industry, their jobs could end up being outsourced. The introduction of the WDS product and its associated practices represents an improvement opportunity for making the depots more competitive with industry.

Depots where senior leadership has understood and capitalized on this important business driver as part of its transition strategy have experienced greater success in transitioning to the WDS product. 


\subsection{Lessons on User Communities}

The lessons learned about user communities are discussed below.

\subsubsection{Create your own user group.}

The MRP II program created its own user group with a regular meeting schedule. This group is intended to help the depots in their implementation and use of the MRP II philosophy. The meetings are typically two days long with the second day open to the vendor. The main advantage of these meetings is that they provide a forum in which the different sites may discuss the difficulties they are having in implementation and the solutions they are using to overcome those difficulties. Because the user group consists of repair depots using MRP II, the problems that one depot encounters are likely to be similar to those that others will encounter. Equally, the solutions that one depot has discovered may be useful to other depots. A further advantage of the MRP II program having its own user group is that the entire group is focused on the business of repairing DoD assets. Thus, issues of DoD policy are also pertinent to all participants.

Having the vendor attend at least a portion of the user group meeting means that the vendor can hear directly from actual users the problems that its DoD customers are having (and may choose to enhance the product to avoid those problems). Additionally, the vendor may provide advice on how its product may be used to overcome the difficulties that the depots are having.

\subsubsection{Join the vendor's user group.}

The MRP II vendor, WDS, runs a user group for all of its customers. The MRP II program office and some of the depots send representatives to these meetings. This user group is valuable as it provides some insight into the use of the product in the commercial sector. Future directions of the product may be discussed in these meetings, providing the DoD with the opportunity to express its needs in the field of remanufacturing.

\subsubsection{Join the appropriate professional and standards bodies.}

The MRP II program uses CompassCONTRACT from WDS, which is based on the business principles embodied in MRP and MRP II. Professional organizations and standards bodies exist to promote and support the MRP II philosophy. Given that the depot's business will be run according to the MRP II principles, it is important that the depots and the program office remain current with the direction being taken by the MRP II philosophy. This can be done through the appropriate professional bodies. Further, by being active in the professional and standards bodies, the DoD is able to influence the future course of the MRP II philosophy so that the business practices embodied by the philosophy are likely to remain more consistent with DoD needs. It should be noted, though, that the DoD can merely influence and cannot control the direction that the MRP II philosophy takes. 


\subsection{Lessons on Deployment}

The lessons learned about deployment are discussed below.

\subsubsection{Data and business processes have to be ready for the COTS product.}

Simply introducing the product does not immediately make life better for the depots. Unless the depots are prepared for the product, they will be unable to take advantage of it. This is particularly true when the product embodies a new way of running the business. The preparation required is to both modify the business processes and ensure that the data is scrubbed and available in a format that can be imported into the product. Without appropriate data, the WDS product will be unable to perform its function. The accuracy of the data will, of course, affect the quality of the product's output.

\subsubsection{The program office is providing guidance rather than enforcing a strict deployment and usage policy.}

The MRP II program office has recognized that there is not "one true way" to use or deploy the WDS product. Given that the different depots operate in different ways, it is important that the depots tailor the product to their needs and even deploy the product the way that they feel is most appropriate.

Different depots have adopted different approaches. For example, the NADEP, Cherry Point has adopted an approach where it takes an entire process, such as shop floor control, from end to end, and deploys that process using the WDS product, regardless of the assets involved. An alternative, taken at the NADEP, Jacksonville, is to deploy the WDS product for a particular workload, such as avionics. Both approaches are equally valid and incrementally lead the depots toward full implementation using the MRP II philosophy.

The program office acts as a guide and, to some extent, as a center of expertise on how the depots can deploy the WDS product. They also act as a clearinghouse, letting depots know what has (and has not) worked at other depots.

\subsubsection{Hiring an experienced professional to help deploy a COTS product is beneficial.}

Some depots were able to use consultants with proven experience in using and deploying MRP II and similar business systems. This experience enabled the depots to leverage proven methods and practices, thus making rapid progress toward full deployment of MRP II. Having an on-site expert in the technology embodied in a COTS product (in this case MRP II) allows a depot to reach solutions to problems more quickly and sometimes provides for inventive ways around those problems. The only danger is that the depot staff may rely on the expert rather than gaining sufficient expertise themselves. Ensuring that crucial expert knowledge is transitioned to appropriate depot staff can minimize this risk. 


\subsubsection{There are advantages to waiting before deploying an upgrade release of a COTS product.}

Like any commercial product, WDS periodically releases upgraded versions of CompassCONTRACT. The MRP II program office works with WDS to understand the nature of changes in each release. That way the program office can determine the probable effects of each release on the depots. Further, the program office can, in some cases, afford to delay deployment of the product until it has seen how the new release performs at other customer sites. All of this data is used by the program office in forming the recommendations to the depots on when to upgrade to the new version. Because the depots are only required to perform one upgrade a year, the instability that occurs from too many version upgrades can be minimized.

\subsubsection{The DoD underestimated the time to transition and deploy MRP II.}

Although the depots are structured such that an order can be carried out efficiently, the transition to using MRP II requires a sequence of steps to be taken. Each of those steps takes time, regardless of the willingness of the participants to hasten the process. It takes time to examine business processes, to change those processes, to prepare data, and to train personnel in the new way of doing business.

This lesson is subtly different from the earlier lesson (2.1.4) concerning business process change in that it encompasses the whole of deployment and not just one aspect[PW1].

\subsubsection{There was insufficient contact with the vendor during deployment.}

During deployment (at the conference room pilots and at other times), some depots found that they had insufficient assistance from the vendor. In part, this may have been a cultural feeling, where the depot staff expected more contact. However, this may also have been caused by the small size of WDS (over the period of its contract with the DoD, it doubled in size in order to handle all of the additional work) as well as limitations in the contract. Including more vendor support in the contract could have avoided this problem. 


\subsection{Lessons on Vendor Relationship Management}

The lessons learned about managing vendor relationships are discussed below.

\subsubsection{Do not buy the source code.}

The MRP II program office started out with, and maintained, a rigid policy of refusing to even try to purchase the source code to the WDS product. The consequences of this policy have, so far, proven to be beneficial to the MRP II program. Specifically, because the source code is not available, there has been no ability to modify the code to meet a particular business process. As a result, the office can accept upgrades from the vendor as they are released and does not need staff to maintain the code.

\subsubsection{Do not ask for DoD-specific modifications to the product.}

The MRP II program office has gone further in terms of keeping a purely COTS-product view of the world. Specifically, they have asked the vendor to make no DoD-specific changes to the product. This means that the depots, as they use the product, are required to use practices in line with the rest of industry — after all, that is what the product supports.

\subsubsection{It is acceptable to influence the vendor when asking for product enhancements.}

The MRP II program office is very careful as to what changes it asks the vendor to make. It is trying to avoid the situation where the vendor is maintaining two copies of the product, one for the DoD and one for industry. WDS has been asked to treat the DoD as it would any other customer. Specifically, if the DoD asks for a particular product enhancement, WDS will make that change only if it makes commercial sense to WDS. One way for the company to check on the viability is to ask its other customers if they would use the enhancement. In this way, the DoD can influence, but not direct, the vendor. 


\section{General Conclusions}

From the very beginning, the MRP II program office has explicitly tried to act as a commercial entity and has encouraged the depots to operate in a business-like way. This has enabled the MRP II program to gain maximum benefit from the COTS products available in the field of remanufacturing. In particular, it meant that the program office and the depots found COTS products that could satisfy their business needs. This contrasts with the circumstance where the DoD defines its own business practices and must acquire customized software, since no commercial products are available to fill all DoD-specific needs.

The emphasis on changing business processes has provided more benefit to the DoD than the COTS product alone. Each repair depot has had the opportunity to reflect on the way in which it does business and to use the introduction of the new product to change inefficient practices. Viewing the product as enabling the repair depots to perform their function (repairing DoD assets) rather than institutionalizing existing practices means that the product assists rather than drives the function of the depots. The emphasis on changing business processes has been coupled with an emphasis on educating and training the depot staff in both the MRP II philosophy and the specific product. This educational emphasis has accelerated progress toward full use of the WDS system.

The decisions not to buy the source code and to use the product as sold rather than with DoDspecific modifications have been essential factors in the success of the MRP II program. These decisions mean that the vendor, WDS, is not making a special version of its product for DoD use. When the DoD acquires COTS products modified specifically for the DoD, there is always the danger that the vendor will have a limited commitment to the product, losing many of the advantages of acquiring COTS products. The MRP II program has avoided this danger by following commercial practice rather than dictating that the new product must operate in some DoD-specific fashion.

The relationship between the program office and WDS is that of customer and vendor rather than the traditional one of government and contractor. This has meant that the depots and WDS can concentrate on their separate businesses (repairing assets and making a profit by selling MRP II technology). This has resulted in a cooperative rather than, as often happens, an adversarial relationship. 


\section{For Additional Information}

To reach us for additional information or assistance, use the contact information below or visit our Internet site at http://www.sei.cmu.edu/cbs.

Lisa Brownsword

Software Engineering Institute

Carnegie Mellon University

1403 Wilson Boulevard, Suite 902

Arlington, VA 22203

Voice: (703) 908-8203

Fax: (703) 908-9317

Email:1lb@sei.cmu.edu
Patrick Place

Software Engineering Institute

Carnegie Mellon University

4500 Fifth Ave

Pittsburgh, PA 15213

Voice: (412) 268-7746

Fax: (412) 268-5758

Email: prp@sei.cmu.edu 



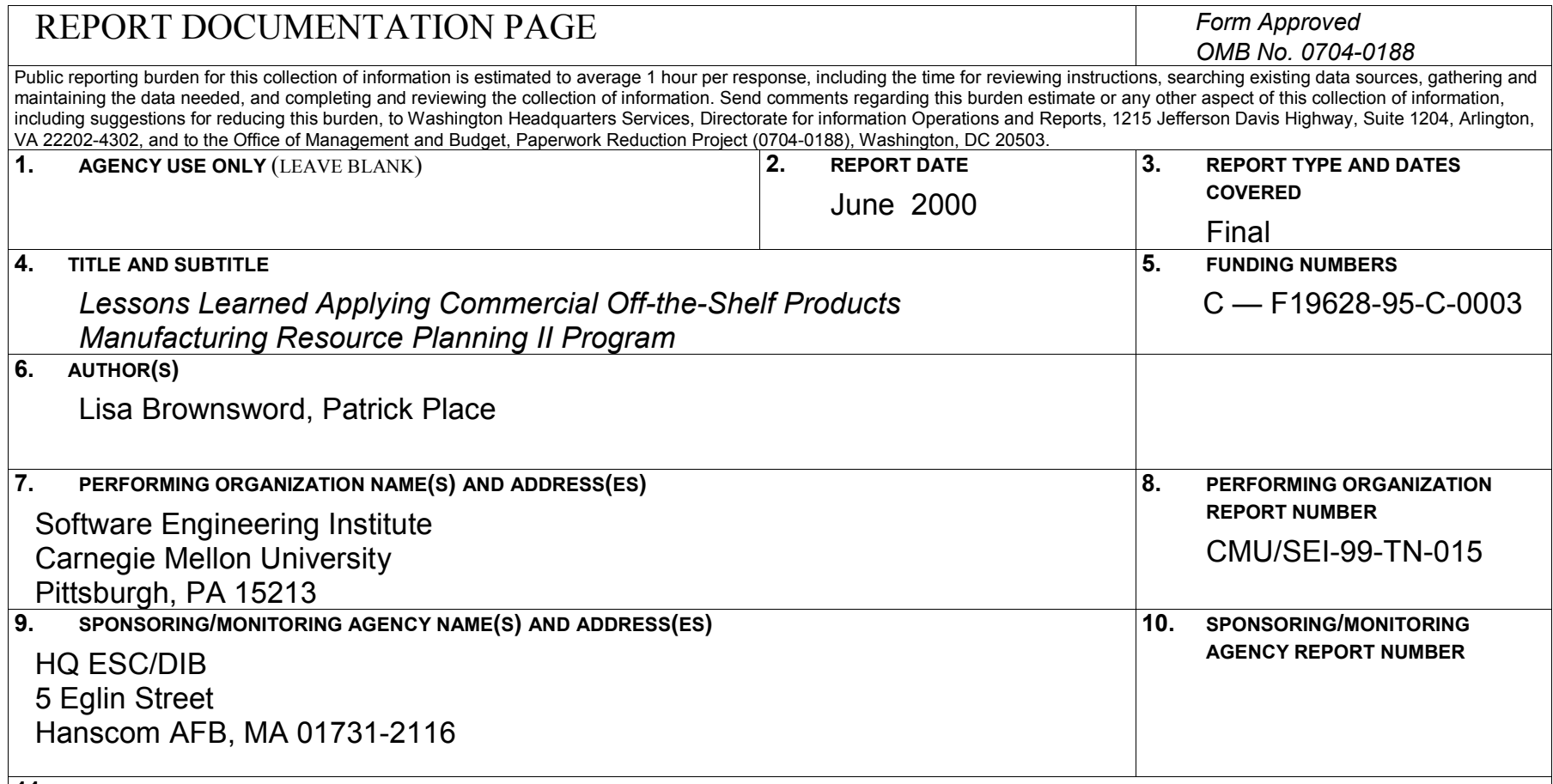

11. SUPPLEMENTARY NOTES

12.A DISTRIBUTION/AVAILABILITY STATEMENT

Unclassified/Unlimited, DTIC, NTIS

13. ABSTRACT (MAXIMUM 200 WORDS)

While the lure of easy system construction from pre-existing building blocks that snap into place is appealing, current reality reveals a less than ideal picture, particularly for commercial off-the-shelf (COTS) software components. Examining the similarities and differences of organizations that have applied COTS and the successes and failures of those organizations has enabled the COTS-Based Systems (CBS) Initiative at the Software Engineering Institute (SEI) to identify a number of significant capabilities that an organization must have to succeed with a COTS-based approach. This case study of the Manufacturing Resource Planning II program is part of a series of case studies that seek to identify important acquisition, business, and engineering issues surrounding the use of COTS-based systems and thus derive available solutions, where possible.

14. SUBJECT TERMS commercial item, COTS, COTS-based systems, project management

\begin{tabular}{l} 
17. SECURITY CLASSIFICATION \\
OF REPORT \\
UNCLASSIFIED \\
\hline NSN 7540-01-280-5500
\end{tabular}

18. SECURITY CLASSIFICATION OF THIS PAGE

UNCLASSIFIED
15. NUMBER OF PAGES 29pp

16. PRICE CoDE

20. LIMITATION OF ABSTRACT

UL

Standard Form 298 (Rev. 2-89) Prescribed by ANSI Std. Z39-18 298-102 